\section{Imaging individual green fluorescent proteins}

Recent advances in fluorescence microscopy techniques have allowed the video-time imaging of single molecules of fluorescent dyes covalently bound to proteins in aqueous environments ${ }^{1}$. However, the techniques have not been exploited fully because proteins can be difficult to label, and dye modification may cause partial or complete loss of activity. These difficulties could be circumvented by fusing proteins to green fluorescent protein (GFP) of the jellyfish Aequorea victoria. Here we report that single S65T mutant GFP molecules ${ }^{2}$ can be imaged using total internal reflection microscopy, and that ATP-driven movement of an individual kinesin molecule (a microtubule motor protein) fused to GFP can be readily observed.

We visualized bacterially expressed GFP or kinesin-GFP fusion proteins adsorbed at a low density to a quartz slide using a total internal reflection (TIR) fluorescence microscope that we had constructed ${ }^{3}$. Circularly polarized light at a wavelength of $488 \mathrm{~nm}$ from an argon laser was focused through a prism to produce total internal reflection and evanescent field illumination of an area roughly $30 \times 40 \mu \mathrm{m}$ on the slide $^{1}$. Fluorescence was collected by an objective lens (Nikon PlanApo 100/1.40), collimated, passed through a customdesigned dichroic mirror and barrier filter (Chroma) and focused onto a chargecoupled device (CCD) camera with a selected intensifier tube (SR UB Gen3+, Stanford Photonics).

There are several reasons to conclude that single GFP molecules are imaged. First, spots disappeared in single quantal events, and not infrequently reappeared at a similar intensity, indicative of reversible photochemistry of a single molecule (Fig. $1)$. Second, the ratio of intensities of GFP spots $(44.1 \pm 1.3$ arbitrary units, mean \pm s.e.m.; $N=182$ ) relative to spots of the dye Cy3 $(51.0 \pm 1.6 ; N=166)$ is 0.86 , as compared to a predicted ratio of 0.90 based on their extinction coefficients at 488 and 514 $\mathrm{nm}$ and the characteristics of our filter sets and camera. Cy3 spots, which show clear single-step photobleaching reactions (Fig. 1 ), have been shown ${ }^{1,4}$ to correspond to single molecules. Third, the fluorescent spot intensities of two fusion products of GFP and motor proteins (kinesin ${ }^{5}$ and $\mathrm{Ncd}^{6}$ ) that were shown to form homodimers by hydrodynamic methods were 1.9 and 2.1 times greater than spots containing GFP fused to a monomeric motor-protein construct (unc104) ${ }^{7}$.

Single GFP molecules showed greater

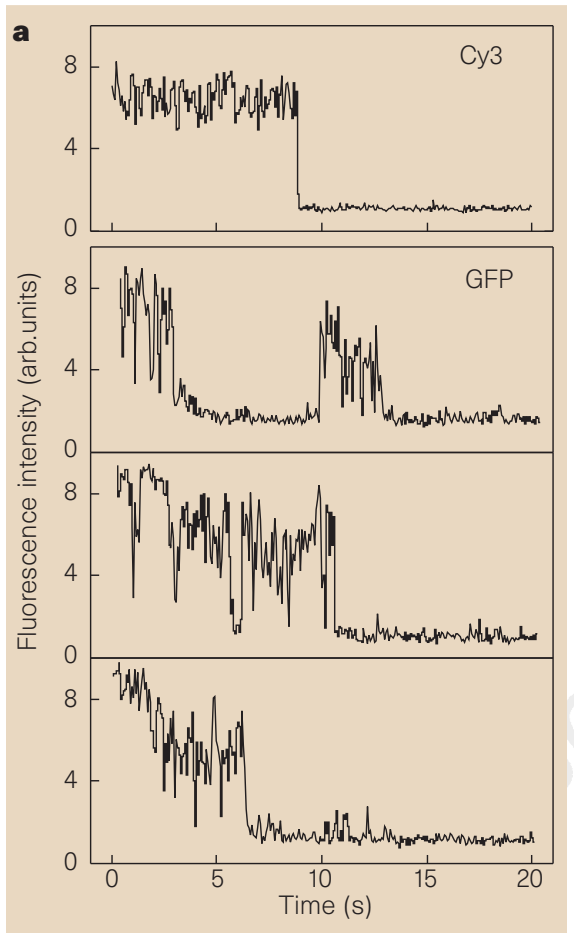

intensity fluctuations than the organic fluorophore $\mathrm{Cy} 3$, and their intensities generally declined during illumination (Fig. 1). This suggests that the protein-embedded chromophore has spectral dynamics that are distinct from organic dyes in solution. Light-driven spectral fluctuations have been observed ${ }^{8}$ for single dye molecules in polymethylmethacrylate films. As dynamics in the core of a protein ${ }^{9}$ may be analogous to those in glassy polymers ${ }^{10}$, we tentatively favour light-driven spectral fluctuations for the origin of the GFP intensity fluctuations.

The disappearance followed by reappearance of fluorescence (Fig. 1) indicates that the chromophore undergoes a reversible reaction. The rate of photobleaching of GFP $\left(0.12 \mathrm{~s}^{-1}\right.$ at $\left.10 \mathrm{~mW}\right)$ is faster than that of Cy3 $\left(0.06 \mathrm{~s}^{-1}\right.$ at $\left.5 \mathrm{~mW}\right)$ and is not influenced by removal of molecular oxygen from the solution. The photobleaching rate of GFP was linearly related to laser power over the available range (2.5-20 mW).

To test the utility of GFP fusion proteins for measuring single molecule dynamics, we fused GFP to residue 560 of the human kinesin heavy chain. We purified the fusion product to homogeneity from an Escherichia coli expression system, and combined it with $1 \mathrm{mM}$ ATP and sperm flagellar axonemes. As described previously for Cy3-labelled kinesin ${ }^{4}$, individual fluorescent spots bound to and moved processively along the axonemal microtubule substrate (Fig. 1). The fluorescence intensities of moving spots indicated that they are single molecules and not aggregates. We also found that kinesin-GFP exhibited

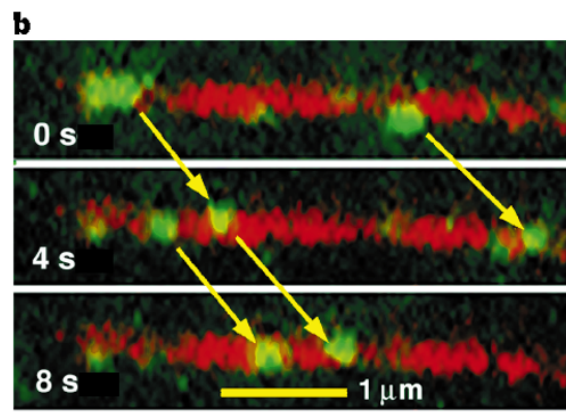

Figure 1a, Single-molecule intensity records for Cy3 dye and GPP (S65T mutant). Typical traces show the intensities of fluorescent spots $(7 \times 7$-pixel area in an 8-bit image, scaled by 1,000) for a kinesinbound Cy3 dye $410 \mathrm{~mW}, 514 \mathrm{~nm}$ illumination) and GFP (20 mW, $488 \mathrm{~nm}$ illumination). Fluorescence from GFP disappears and reappears abruptly. There is a higher noise level (at comparable signal level) of GFP compared with Cy3, and the GPP fluorescence intensity declines after illumination. b, Individual kinesin-GPP molecules (pseudo-coloured green) moving along Cy5-labelled axonemal microtubules (pseudo-coloured red) in the presence of $1 \mathrm{mM}$ ATP and previously described assay conditions ${ }^{4}$

microtubule-based motility when expressed by in vitro transcription and translation in rabbit reticulocyte lysate or wheat-germ extract, and diluted into an ATP-containing buffer.

These GFP assays have advantages over our previously published Cy3 assay ${ }^{4}$ in ease as well as having a stoichiometric amount of fluorophore at a known position. We have extended our method to other motors that are difficult to purify and label by chemical modification.

The ability to measure kinesin-GFP molecule dynamics in real time also suggests broader applications of the technique. It may be possible to study single molecule dynamics of many types of proteins or protein complexes, particularly ones that are difficult to label by chemical means. Our preliminary data indicate that single molecules of GFP fused to membrane or juxtamembrane proteins can be imaged in living cells.

Daniel W. Pierce

Nora Hom-Booher

Ronald D. Vale

Howard Hughes Medical Institute and Department of Pharmacology,

University of California, San Francisco,

California 94143-0450, USA

e-mail:vale@phy.ucsf.edu

\footnotetext{
1. Funatsu, T., Harada, Y., Tokunaga, M., Saito, K. \& Yanagida, Y. Nature 374, 555-559 (1995).

2. Heim, R., Cubitt, A. B. \& Tsien, R. Y. Nature 373, 663-664 (1995).

3. Pierce, D. W. \& Vale, R. D. Methods Cell Biol. (in the press).

4. Vale, R. D. et al. Nature 380, 451-453 (1996).

5. Navone, F. et al. J. Cell Biol. 117, 1263-1275 (1992).

6. McDonald, H. B. \& Goldstein, L. S. Cell 61, 991-1000 (1990).

Otsuka, A. J. et al. Neuron 6, 113-122 (1991)

8. Lu, H. P. \& Xie, X. S. Nature 385, 143-146 (1997).

9. Ormo, M. et al. Science 273, 1392-1395 (1996)

10. Pierce, D. W. \& Boxer, S. G. J. Phys. Chem. 96, 5560-5566 (1992).
} 